\title{
Particle versus Field Structure in Conformal Quantum Field Theories
}

\author{
Bert Schroer \\ presently CBPF, Rua Dr. Xavier Sigaud, 22290-180 Rio de Janeiro, Brazil \\ email: schroer@cbpf.br \\ Prof. emeritus of the Institut für Theoretische Physik \\ FU-Berlin, Arnimallee 14, 14195 Berlin, Germany
}

May 9, 2000

\begin{abstract}
I show that a particle structure in conformal field theory is incompatible with interactions. As a substitute one has particle-like exitations whose interpolating fields have in addition to their canonical dimension an anomalous contribution. The spectra of anomalous dimension is given in terms of the Lorentz invariant quadratic invariant (compact mass operator) of a conformal generator $R_{\mu}$ with pure discrete spectrum. The perturbative reading of $R_{0}$ as a Hamiltonian in its own right i.e. associated with an action in a functional integral setting naturally leads to the AdS formulation. The formal service role of AdS in order to access CQFT by a standard perturbative formalism (without being forced to understand first massive theories and then taking their scale-invariant limit) vastly increases the realm of conventionally accessible 4-dim. CQFT beyond those for which one had to use Lagrangians with supersymmetry in order to have a vanishing Beta-function.
\end{abstract}

\section{A few introductory remarks}

Ideas about the use of conformal quantum field theory entered particle physics for the first time at the height of the Kramers-Kronig dispersion relations [1]. They were met with reactions ranging from doubts to outright rejection and the subject lay dormant for another 10 years when it reemerged on the statistical mechanics side in connection with second order phase transitions.

In the next section we will show that these early doubts of the old-time particle physicists were partially justified, because the particle structure in CQFT is indeed incompatible with interactions. However far from supplying a coffin nail for its utility in high energy physics, this no-go theorem also contains the message that one must use finer concepts in order preserve the usefulness of conformal quantum field theory as a theoretical laboratory for particle physics. 
There are massive particle-like objects ("infraparticles" [3]) which have a continuous mass distribution with an accumulation of spectral weight at $p^{2}=m^{2}$ whose generating local fields have an anomalous non-integer (non-semi-integer in the case of Fermion fields) contribution to their long distance behavior. In a CQFT long and short distance behavior coalesce and the accumulation of spectral weight at $p^{2}=0$ which becomes related to the anomalous dimension of operators is the vestige of the particle interaction in the massive parent theory from which the CQFT arose by taking the scale-invariant limit. This structure is the collective effect of a total collapse of all multiparticle thresholds on top of each other. The standard LSZ large time scattering limit does not commute with this scaling limit, in fact the LSZ limit of such fields vanishes. It is believed that in order to re-extract from such a situation anything which resembles particle physics one has to apply a more general form of scattering theory [3] which is based on expectation values and probabilities for inclusive cross sections (where outcoming "stuff" below a prescribed energy-momentum resolution is not registered) rather than on amplitudes. But it is presently not clear how one can achieve this. In the case of infraparticles (the electron in QED which is inexorably linked to its photon-cloud) where one also meete a situation of coalescing thresholds, this generalized scattering theory is known to be very useful [3].

Recently there has been a quite different and conceptually less ambitious but formally quite attractive idea which promises to strengthen the utility of CQFT for particle physics and which is presented in the third section. It basically consists in finding a theory which radically reprocesses the spacetime interpretation and degrees of freedom of CQFT in such a way that now the "energy momentum vector" $R_{\mu}$ of the Dirac-Weyl compactified world $\bar{M}$ becomes the bona fide energy momentum instead of $P_{\mu}$ which in standard canonical or functional terminology means that $R_{\mu}$ is the one related to an action and not $P_{\mu}$. If one insists that this total reshuffling of physical interpretation should leave the basic mathematical building blocks (a certain generating set of algebras and the symmetry group structure) untouched, then there is only one answer: an associated anti De Sitter (AdS) theory [2]. The nontrivial reprocessing leads to a mathematical isomorphism as described in [- i.e. it goes far beyond that picture about the AdS-CQFT correspondence which is limited to the (infinitely remote) boundary of AdS (see in particular the remarks at the end of [5). The AdS appearance of the AdS structure as a kind of reprocessed CQFT is less surprizing if one recalls the 6-dimensional lightcone formalism which one uses in order to obtain an efficient description of the conformal compactification $\bar{M}$ of Minkowski space $M$ and the construction of its covering $\tilde{M}[9]$.

In this way one obtains a (perturbative) new constructive non-Lagrangian access to CQFT which opens a new window into the realm of CQFT beyond those few 4-dimensional Lagrangian candidates for which one had to use a combination of gauge theory with supersymmetry. This means that one has no

\footnotetext{
${ }^{1}$ The attribute "conceptually" here refers to the local quantum physical aspects and not to differential-geometric ones.
} 
guaranty that the conformal side at all permits a description in terms of an action.

\section{Particle Structure and Triviality}

We start with recalling an old theorem which clarifies the relation between the particle-versus-field content of conformal field theories. To be more precise the following statement is a result of the adaptation of a combination of several theorems 6]

Theorem 1 The existence of one-particle states in conformally invariant theories forces the associated interpolating fields to be canonical free fields. The only particle-like structures consistent with interactions are hidden in the structure of those interpolating fields which have anomalous dimensions and whose mass spectrum is continuous with an accumulation of weight at $p^{2}=0, p_{0}>0$.

The easiest way to get a first glimpse at this situation is to look at conformal two-point functions

$$
\left\langle\psi(x) \psi^{*}(y)\right\rangle=\left\{\begin{array}{c}
c \frac{1}{-(x-y)^{2}}, \operatorname{dim} \psi=1 \\
c\left(\frac{1}{-(x-y)^{2}}\right)^{d_{\psi}}, \operatorname{dim} \psi=d_{\psi}>1
\end{array}\right.
$$

In the first case the application of the LSZ large time scattering limit yields

$$
\left\langle\psi(x) \psi^{*}(y)\right\rangle=\left\langle\psi_{\text {in }}(x) \psi_{i n}^{*}(y)\right\rangle
$$

which preempts the equality $\psi=\psi^{\text {in }}=\psi^{\text {out }}$, whereas in the anomalous case the large distance fall-off is too strong in order to be reconcilable with the mass shell structure of a zero mass particle which means

$$
\psi(x) \stackrel{L S Z}{\rightarrow} 0
$$

It is worthwhile to reconsider the argument which leads to the absence of interaction in the space created by the interpolating field $\psi$. The crucial observation is that the presence of a zero mass scalar particle state vector $|p\rangle$ with

$$
\langle p|\psi| 0\rangle \neq 0
$$

forces $\psi$ to have a two-point function with a canonical scale dimension $\operatorname{dim} \psi=1$. The special feature of conformal invariance is that this implies that the two-point function is free i.e.

$$
\left\langle 0\left|\psi^{*}(x) \psi(y)\right|\right\rangle=c \frac{1}{[-(x-y-i \varepsilon)]^{2}}
$$

Such a conclusion relating canonical short distance dimension with absence of interactions cannot be drawn in the massive case. However the following theorem which was proven in the late $50^{i e s}$ by Jost and the present authors, and can be found in 8 , holds for both cases: 
Theorem 2 The freeness of the $\psi$ two-point function implies the field $\psi$ to be a free field in Fock space.

The guiding idea is to show that a localized operator or pointlike field which vanishes on the vacuum, vanishes automatically on all states i.e. is the zero operator. This is a consequence of the Reeh-Schlieder theorem [8] which in conformal field theory is also known under the name state-field relation). It says that the operators from a region with a nontrivial causal complement (or fields smeared with test functions with support in such a region) act cyclically on the vacuum (and on any other finite energy state). If we denote by $\mathcal{A}(\mathcal{O})$ either the polynomial *-algebra of unbounded smeared fields with supports of testfunctions in $\mathcal{O}$ or the affiliated bounded operator algebra, this cyclicity property reads

$$
\overline{\mathcal{A}(\mathcal{O}) \Omega}=\mathcal{H}
$$

where the bar denotes the closure and $H$ is the Hilbert space generated by all fields (bosonic and fermionic). Since (for fermionic $\psi$ there will be a change of sign)

$$
\psi(x) \mathcal{A}(\mathcal{O}) \Omega=\mathcal{A}(\mathcal{O}) \psi(x) \Omega
$$

if we choose $O$ spacelike with respect to $x$, the vanishing of the "current" $j(x)=$ $\left(\partial_{\mu} \partial^{\mu}+m^{2}\right) \psi(x)$ on the vacuum implies the vanishing on the dense set $\mathcal{A}(\mathcal{O}) \Omega$ and hence (operators in physics are closable) on all $\mathcal{H}$. The next step consists in proving that the commutator of two $\psi s$ on the vacuum is a c-number

$$
([\psi(x), \psi(y)]-i \Delta(x-y)) \Omega=0
$$

It then follows according to the previous argument that the bracket vanishes identically. We prove this last relation by using the frequency decomposition $\psi=\psi^{(-)}+\psi^{(+)}$(which follows from $j \equiv 0$ ) in the commutator

$$
[\psi(x), \psi(y)] \Omega=\left(\left[\psi^{(+)}(x), \psi^{(+)}(y)\right]+\psi^{(-)}(x), \psi^{(+)}(y)-\psi^{(-)}(y), \psi^{(+)}(x)\right) \Omega
$$

where we omitted all annihilation terms. The on-shell creation with subsequent on-shell annihilation as in the last two terms and the physical spectrum condition only admits the vacuum as its energy momentum content and therefore they yield a c-number which, by a finite renormalization of $\psi$ if necessary, yields

$$
\left(\psi^{(-)}(x), \psi^{(+)}(y)-\psi^{(-)}(y), \psi^{(+)}(x)\right) \Omega=i \Delta(x-y) \mathbf{1} \Omega
$$

Since this and the full commutator is causal, the first term on the right hand side has to vanish all by itself. But on the other hand it is the separate Fouriertransform of momenta which lie on the forward mass shell and hence it is the boundary value of an analytic function in two complex 4 -vectors of the form $z=\xi-i \eta, \eta$ from the forward light cone. However an analytic function which vanish on an open set on its boundary vanished identically (generalized 
Schwartz reflection principle). The resulting relation on the vacuum holds according to the previous arguments for the operators and therefore we obtained the characterizing relation for a free field. The generalization to any spin including half-integer values is now a routine matter. A closer look at the zero mass situation reveals that contrary to the massive case where the difference of two on-shell vectors is either spacelike or zero, the difference of two lightlike vectors may in addition be lightlike but this only happens for parallel vectors. Since this special configurations should not matter in the sense of $\mathrm{L}^{2}$-integrability of zero mass particle wave functions one again expects at least for $d>1+1$ the above result. However a mathematical proof of this result turned out to be quite nontrivial [7].

It is very helpful to place the above theorem into the setting of a more general theorem relating interactions and particle properties in general local quantum physics which states that operators localized in sub-wedge regions in interacting theories which possess nontrivial matrix elements between vacuum and one-particle states necessarily show the phenomenon of vacuum polarization i.e. operators which create polarization-free one-particle states exist only in interaction free field theories. Polarization-free-generators (PFG) which create pure one-particle states from the vacuum do however exist in any QFT if their localization region is a semi-infinite wedge region or larger [10]11]. Since in conformal theories the wedge region is conformally equivalent to a compact double cone, a conformal one-particle structure according to this more general theorem is only possible in conformal free field theories.

The above argument is typical for a real-time structure which cannot be unraveled in the euclidean formulation.

\section{$3 \quad$ Trying to make the best out of it}

The negative result on the compatibility of zero mass particle structure with nontriviality of conformal theories should not be misread as an incompatibility with an intuitive idea about what constitutes particle-like excitations. The point here is that conformal theories in particle physics should be considered as the zero mass (scaling) limits of massive theories with mass gaps for which the LSZ scattering theory can be derived. In the scaling limit all the multiparticle thresholds in momentum space coalesce on top of each other and build up the possibly anomalous dimension. In this limit the Wigner particle theory (irreducible representation of the Poincaré group) and with it the prerequisite of the LSZ scattering theory gets lost in the presence of interactions, a fact which we have demonstrated above where it was shown that the field is either free or the LSZ limits are zero. So the right question would be: can one think of a more general scattering theory which may recuperate some of the lost structure in the aforementioned collapse of multiparticle cuts on top of each other? There is indeed another particle concept ("infraparticle") which goes together with a generalized scattering theory build on inclusive scattering probabilities instead of amplitudes [3]. This concept is expected to distinguish those anomalous di- 
mensional fields which are of relevance in particle physics (which originate from the previous collapse in the scaling limit) from mere mathematical constructs as e.g. generalized free fields with anomalous dimensions. But we think that for the problem at hand, namely the formulation of a theory of anomalous dimension, we do not need to enter this deep and difficult issue of particle-like interpretation since here we restrict our interests in conformal theories as a simplified theoretical laboratory for field- and algebra- aspects and not for the study of particles and their scattering theory. We believe that the setting of local observable algebras which fulfill in addition to Einstein causality also Huygens principle for timelike distances 12] contains all scale limits of theories which are of interest for particle physics and that interaction in this setting is characterized by the appearance of charge-carrying fields with anomalous dimensions. In view of the above No-Go theorem we will consider the noncanonical (anomalous dimension) nature of those fields as our pragmatic definition of interaction in this conformal setting. But we defer this analysis to a following longer paper which contains the relevant mathematical machinery [12].

As a consequence the observable algebra of an interacting conformal field theory (conserved currents etc.) should not have the structure of composites of free fields (e.g. free currents) since otherwise the fields carrying the superselected charges may not have anomalous dimensions. Apart from normalization constants the 2- and 3-point functions of conformal observable fields (currents) are indistinguishable from those formed with free composites with the same integer dimensions. If all correlations would be indistinguishable from those of free composites (total protection) then also the charge-carrying fields associated with such observables can be shown to be free.

A weak form of what in the case of conformal SYM theories has been called (partial) "protection" would be one where the relative normalization between 2-and 3-point functions is that of free composites (partial protection). Apparently perturbative supersymmetry causes partial protections [13]. Although such models hardly represent realistic particle physics, they are the only $L a$ grangian candidates for $\mathrm{d}=1+3$ nontrivial conformal field theories and may yet turn out to be the first 4-dimensional mathematically completely controllable models. The interest and fascination in conformal field theories originates to a large part from the well-founded belief that the simplest nontrivial 4-dimensional conformal field theories which will break the age old existence deadlock for nontrivial quantum field theories in physical spacetime. For this one wants to have as much protection as possible without ending with a free conformal theory.

Instead of entering an ambitious program in order to extract the particle physics "honey" from CQFT which requires a heavy conceptual investment in the area of a generalized scattering theory, there is another way which is more faithful to the formal aspects with which QFT is often identified (erroneously

\footnotetext{
${ }^{2}$ In any area of Theoretical Physics there always have been plenty of nontrivial mathematically controllable illustrations which demonstrate the nontrivial physical content of the conceptual basis of those areas, not so in 4-dim. QFT. This annoying totally singular situation has been sometimes overemphasized at the cost of practical calculations, but most of the time it went totally ignored.
} 
in my opinion, if one uses them for a definition of QFT) namely canonical formalism and/or functional integrals. It starts from the observation that in addition to the translation generator $P_{\mu}$ there is another translation-analogue described by a Lorentz-vector $R_{\mu}$. It has a timelike purely discrete spectrum and the L-invariant "mass" $m_{c}$ with $m_{c}^{2}=R_{\mu} R^{\mu}$ plays a similar role as the rigid rotation operator $L_{0}$ in chiral theories. In fact it describes a generalized rotation around the Dirac-Weyl compactified Minkowski space $\bar{M} \simeq S^{3} \times S^{1}$. Therefore it is not surprising that the bottom of the spectrum of $m_{c}$ is the anomalous part of the scaling dimension common to a whole equivalence class of fields which carry the same superselected charge. But despite all analogies to $P_{\mu}$ this operator is not related to an imagined functional integral action of CQFT. Nevertheless one can ask the question: is there a theory whose Lagrangian can be associated with a Hamiltonian interpretation of $R_{0}$ ? In order for this new theory to be useful for particle physics it should keep the same algebraic and group-theoretical building blocks as CQFT i.e. one seeks a mathematical isomorphism which goes hand in hand with that total physical reprocessing which is necessary to accomplish such an impossible looking task. The unique answer is the AdS-CQFT correspondence [2] which was proven to be a such a "radical" isomorphism [4.

Although this step does not completely answer the question posed at the beginning of how to extract and analyze the particle content of CQFT, it goes a long way to open up conformal field theory as a genuine theoretical laboratory for particle physics. And last not least it facilitates the unsolved problem number one: find a nontrivial physically relevant (i.e. one which fits at least the conceptual framework of local quantum physics, even if it falls short in describing nature) and mathematically controllable model in 4-dimensional QFT.

The presented arguments suggest strongly that there exists a whole world of non-Lagrangian non-supersymmetric CQFT (in the sense that they cannot be accessed in the standard perturbative way) besides the Lagrangian SYM family. In fact the perturbative calculations in the literature already give some support in this direction. This is most visible in 14 although these authors, evidently under the strong spell of the string-theoretic origin of the AdS-CQFT, do not interprete their calculations from this viewpoint.

The possible non-Lagrangian nature of most CQFT is in a certain way explained by Rehren's deep observation [4] [ that due to the isomorphic nature of the AdS-CQFT relation there must be degrees of freedom on the conformal side which cannot be described in terms of local fields namely those which originate from the AdS bulk (and not from the boundary) and which are necessary in order to return $C Q F T \rightarrow A d S$. This leaves the interesting question of what should one make of the original observation by which the protagonists of the AdS-CQFT correspondence found this relation which is the relation between two Lagrangian field theories namely the conformal SYM model with some form of AdS supergravity [2]. Since this is based on consistency checks within string theory which owes its widespread acceptance to perturbative mathematical consistency and a kind of globalized social contract but certainly not to its harmonious coexistence with the principles underlying particle physics, there is 
reason for some scepticism; in particular because such degrees of freedom would be easily overlooked in perturbative calculations on the CQFT side. It cannot be overstressed that this correspondence is very different and much more radical then those which arise from a different choice of "field coordinates". It is impossible to understand its full content in terms of pointlike physical fields.

\section{Some concluding remarks}

If, as argued in this letter, the AdS theories are a useful new calculational tool which open up CQFT to particle physics studies within the standard Lagrangian quantization framework, than perhaps with an additional conceptual investment one could directly understand the structure underlying the anomalous dimension spectra within CQFT i.e. without the described reprocessing on the AdS side. This turns out to be true and will be the subject of a subsequent paper [12] since the necessary conceptual investment does not fit the format of a letter like this.

Acknowledgements: I am indebted to Detlev Buchholz and Karl-Henning Rehren for a helpful exchange of emails. Furthermore I would like to thank Francesco Toppan for interesting questions which helped in shaping the presentation.

\section{References}

[1] H.A. Kastrup, Ann. Physik 7, (1962) 388

[2] J. Maldacena, Adv. Theor. Math. Phys. 2 (1998), 231

S.S. Gubser, I. R. Klebanov and A.M. Polyakov, Phys. Lett. B448, (1998) 253

E. Witten, Adv. Theor. Math. Phys. 2 (1998) 253

[3] D. Buchholz, "Mathematical Physics Towards the 21st Century", Proceedings Beer-Sheva 1993, Ben Gurion University Press 1994

[4] K-H Rehren, "Algebraic Holography", hep-th/9905179

[5] K-H Rehren, "Local Quantum Observables in the Anti-deSitter - Conformal QFT Correspondence", hepth/0003120

[6] D. Buchholz and K. Fredenhagen, JMP 18, Vol.5 (1977) 1107

[7] K. Pohlmeyer, Commun. Math. Phys. 12, (1969) 201

[8] R.F. Streater and A.S. Wightman, PCT, Spin and Statistics and all That, Benjamin 1964

[9] B. Schroer, "Facts and Fictions about Anti de Sitter Spacetimes with Local Quantum Matter", hep-th/9911100 
[10] B. Schroer, "Particle Physics and QFT at the Turn of the Century: Old principles with new concepts, (an essay on local quantum physics)", Invited contribution to the Issue 2000 of JMP, in print, to appear in the June issue

[11] H-J Borchers, D. Buchholz and B. Schroer, "Polarization-Free Generators and the S-Matrix", hep-th/0003243

[12] B. Schroer, "A Theory of Anomalous Scale-Dimensions", hep-th/0005134

[13] for example: J. Erdmenger, M. Perez-Victoria, "Non-renormalization of next-to-extremal correlators in $\mathrm{N}=4 \mathrm{SYM}$ and the AdS/CFT correspondence" and literature quoted therein

[14] L. Hoffmann, A.C. Petkou and W. Ruehl, "Aspects of Conformal Operator Product Expansion in AdS/CFT Correspondence", hep-th/0002154 